\title{
Utilização do ZEBMAX® para Aumento de Produtividade na Alface
}

\author{
Use of ZEBMAX® to Increase Lettuce Productivity
}

\author{
Emmanuel Zullo Godinho*a; Hélio Vagner Gasparotto; Fernando de Lima Caneppele ${ }^{\mathrm{b}}$
}

aUniversidade Estadual Paulista "Júlio de Mesquita Filho”. SP, Brasil.

bUniversidade de São Paulo, Faculdade de Zootecnia e Engenharia de Alimentos. SP, Brasil.

*E-mail: emmanuel.godinho@unesp.br

\begin{abstract}
Resumo
Dentre as hortaliças cultivadas a Alface (Lactuca sativa) destaca-se devido a sua fonte de vitaminas $\mathrm{B}_{1}, \mathrm{~B}_{2}$, C, ferro e minerais, além da disponibilidade de ser cultivada em quase todas as regiões do globo terrestre. Diante do exposto, o objetivo do trabalho foi avaliar, em uma cultura de alface, a massa fresca de parte aérea e massa fresca da raiz com aplicação do ZEBMAX®. O experimento foi realizado à campo no Colégio Agrícola de Toledo no período de 10 de junho a 15 de julho de 2018 . No tratamento foram realizadas aplicações de duas doses de 0,5 $\mathrm{ml} \mathrm{L}^{-1}$ aos 15 e 25 dias após o transplantio. Já na testemunha só foi utilizada água. Utilizou-se o delineamento de blocos ao acaso em triplicata e no total foram avaliadas 10 plantas por bloco. Os dados foram submetidos às análises estatísticas usando o teste de Tukey, ao nível de $5 \%$ de probabilidade. As plantas do tratamento obtiveram um peso de massa fresca de parte aérea de $12,55 \mathrm{~g} \mathrm{pl}^{-1}$, com a massa fresca de raiz de $10,88 \mathrm{~g} \mathrm{pl}^{-1}$, já na testemunha o peso da massa fresca de parte aérea ficou 11,08 $\mathrm{g} \mathrm{pl}^{-1}$ com a massa fresca de raiz 9,44 $\mathrm{g} \mathrm{pl}^{-1}$. Portanto, o presente trabalho apresentou diferenças significativas para as variáveis de crescimento (raiz e da parte aérea), em relação a aplicação do ZEBMAX® como fonte de adubação, na relação entre o tratamento e a testemunha.
\end{abstract}

Palavras-chave: Massa Fresca de Parte Aérea. Massa Fresca de Raiz. Peso de Planta.

\begin{abstract}
Among the vegetables grown, Lettuce (Lactuca sativa) stands out due to its source of vitamins $B_{1}, B_{2}$, C, iron and minerals, in addition to the availability of being grown in almost all regions of the globe. Given the above, the objective of the work was to evaluate, in a lettuce culture, the fresh mass of aerial part and fresh mass of the root with the application of the ZEBMAX®. The experiment was carried out in the field at the Colégio Agricola de Toledo from June 10 to July 15, 2018. In the treatment, two doses of $0.5 \mathrm{ml} \mathrm{L} \mathrm{L}^{-1}$ were applied at 15 and 25 days after transplanting. In the witness, only water was used. A randomized block design in triplicate was used and a total of 10 plants per block were evaluated. The data were submitted to statistical analysis using the Tukey test, at the level of 5\% probability. The treatment plants obtained a weight of fresh mass of aerial part of $12.55 \mathrm{~g} \mathrm{pl}^{-1}$, with a fresh root mass of $10.88 \mathrm{~g} \mathrm{pl}^{-1}$, while in the control the weight of fresh mass of aerial part was $11.08 \mathrm{~g} \mathrm{pl}^{-1}$ with fresh root mass $9.44 \mathrm{~g} \mathrm{pl}^{-1}$. Therefore, the present study showed significant differences for the growth variables (root and shoot), in relation to the application of ZEBMAX® as a source of fertilization, in the relationship between the treatment and the control.
\end{abstract}

Keywords: Fresh Mass of Aerial Part. Fresh Root Pasta. Plant Weight.

\section{Introdução}

Observando-se dados do PIB brasileiro, o setor agro se destaca perante outros setores da economia, dentro desse setor a área as olericulturas apresentam crescimento exponencial, principalmente a produção de folhosas, como a alface (BRASILHORTIFRUTI, 2020).

Os principais produtores dessa hortaliça são os paulistas e os mineiros (SALA; COSTA, 2012), sendo que, na safra 2018/2019 esta folhosa teve uma área plantada de 15.300 hectares e uma produção de aproximadamente sete mil toneladas da variedade crespa (BRASILHORTIFRUTI, 2019).

Menezes Júnior et al. (2004), colocaram que, as hortaliças, no geral, apresentam grande exigência nutricional para que haja seu desenvolvimento, principalmente em relação aos micronutrientes. A alface (Lactuca sativa L.) é considerada a melhor variedade para o plantio em climas amenos, principalmente no inverno e outono no Brasil, além de ser a cultura folhosa mais consumida e admirada no país, principalmente nas regiões sul e sudeste (BLAT et al., 2011).

Além dos fatores climáticos, as novas tecnologias implantadas também estão influenciando as mudanças na produção brasileira, principalmente produtos que são aplicados com constituição de baixo impacto no meio ambiente (ZIECH et al., 2014). Sabe-se que desde as pequenas a grandes culturas utilizam macro e micronutrientes para aumento de produtividade, muitas vezes estes produtos aplicados via foliar e são complexos de diversos nutrientes (RESENDE et al., 2010).

Estes produtos são muitas vezes aplicados para suprir uma falta de nutrientes nas plantas, ou até mesmo utilizando como produtos preventivos a ataques de pragas e doenças, Godinho et al. (2019), apresenta bons resultados em olerícolas com a aplicação de produtos direcionados para seu estágio de desenvolvimento. 
Diante do exposto, o objetivo do trabalho foi avaliar, em uma cultura de alface, a massa fresca de parte aérea e massa fresca da raiz com aplicação do ZEBMAX®.

\section{Material e Métodos}

$\mathrm{O}$ experimento foi conduzido à campo no Colégio Agrícola de Toledo, de Latitude Sul 24 47' 16" e Longitude Oeste $53^{\circ} 43^{\prime}$ '29'. Foi semeado em bandeja de isopor com 200 células utilizando o substrato Carolina Soil ${ }$. Após 15 dias realizou-se o transplantio e na sequência, feitas duas aplicações do produto ZEBMAX® (P 20\%; S 1\%; Cu 1\%; Mn 1\%; N 3\%), sendo aos 15 e 25 dias após o transplantio nos canteiros. Ambas as aplicações tiveram dosagens de 20 $\mathrm{ml} \mathrm{L} \mathrm{L}^{-1}$. A condução do experimento ocorreu em delineamento experimental em blocos ao acaso com três repetições, sendo avaliadas seis plantas por linha, totalizando 36 plantas. As aplicações do fertilizante foram realizadas por meio de um borrifador de $1 \mathrm{~L}$.

Para o tratamento controle foi utilizada água. As parcelas experimentais constituíram-se de canteiros com seis linhas de 3,5 m de comprimento por 2,5 m de largura, com uma área de $8,75 \mathrm{~m}^{2}$, com espaçamento entre linhas de $0,30 \mathrm{~m}$ e entre plantas de 0,30 m (Figura 1).

Figura 1 - Croqui da área de plantio da alface cv. roxo

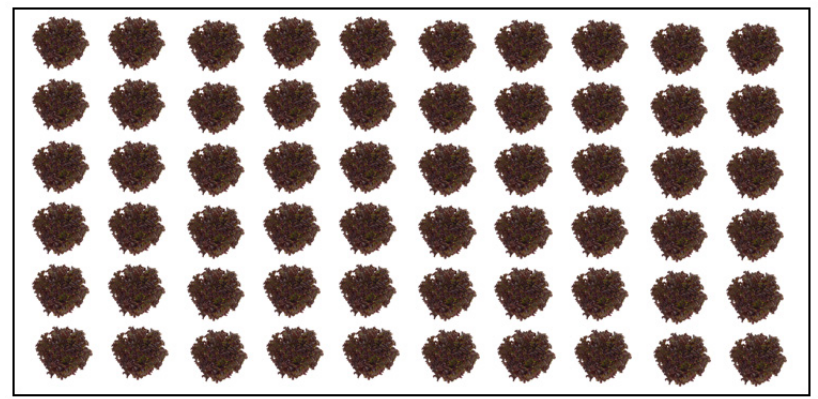

Fonte: Os autores.

Os canteiros (tratamento e testemunha) foram cobertos com maravalha afim de controlar as plantas daninhas e manter o solo hidratado. A metodologia usada neste trabalho foi descrita e adaptada por Resende et al. (2003). Foi utilizado uma balança de precisão com 8 dígitos para a pesagem dos materiais. Após a colheita, as folhas foram separadas das raízes para pesagem das amostras. Com isso foi avaliado o peso da massa fresca de parte aérea e massa fresca de raiz em grama por planta $\mathrm{g} \mathrm{pl}^{-1}$. Os dados foram submetidos à análise de variância e comparados pelo teste de Tukey e AlexandreDarling ao nível de 0,05 com auxílio do programa estatístico Action $^{\mathrm{TM}}$ e para os gráficos foi usado o Origin 6.0.

\section{Resultados e Discussão}

No ensaio de massa fresca da parte aérea houve um aumento significativo, com uma média geral no tratamento de $12,55^{\mathrm{a}} \mathrm{em} \mathrm{g} \mathrm{pl}^{-1}$, na testemunha de $11,08^{\mathrm{b}}$, o seu $\mathrm{p}<0,05$. $\mathrm{Na}$ massa fresca de raiz o resultado final no tratamento foi de $10,88^{\mathrm{a}}$, já na testemunha $9,44^{\mathrm{b}}, \mathrm{o} \mathrm{p}<0,05$. Aplicado o teste de
Tukey a 5\% de probabilidade, os resultados tiveram diferença significativa entre si, conforme apresentado na ANOVA (Quadro 1).

Quadro 1 - Rendimento de massa fresca de parte aérea e massa fresca de raiz, em função da aplicação do fertilizante foliar ZEBMAX®

\begin{tabular}{|l|c|c|}
\hline & MFPA $\left(\mathbf{g ~ p l}^{-\mathbf{1}}\right)$ & \left. MFR ${\mathbf{~} \mathbf{~} \mathbf{~ p}^{\mathbf{1}}}^{\mathbf{1}}\right)$ \\
\hline Tratamento & $12,55^{\mathrm{a}}$ & $10,88^{\mathrm{a}}$ \\
\hline Testemunha & $11,08^{\mathrm{b}}$ & $9,44^{\mathrm{b}}$ \\
\hline Média & 11,82 & 10,16 \\
\hline CV (\%) & 1,16 & 7,67 \\
\hline p-valor & 0,000000007 & 0,00000002 \\
\hline Anderson-Darling & $0,4113^{*}$ & $0,2053^{* *}$ \\
\hline
\end{tabular}

MFPA: massa fresca de parte aérea $\left(\mathrm{g} \mathrm{pl}^{-1}\right)$; MFR: massa fresca de raiz $(\mathrm{g}$ $\left.\mathrm{pl}^{-1}\right) ; \mathbf{C V}(\%)$ : coeficiente de variação. $\mathbf{A D}^{*}$ : p-valor AD 0,2739; $\mathbf{A D}^{* *}$ : p-valor AD 0,2053

Fonte: Dados da pesquisa

Reforçando os resultados de CV, Ferreira (2018) coloca que, CV (\%) menor ou igual que 15\%, resulta em uma baixa dispersão, ou seja, os dados encontrados são considerados homogêneos, na MFPA o CV (\%) foi de 1,16 e na MFR 3,28.

De acordo com Bündschen et al., (2018a), ao aplicar um fertilizante foliar na rúcula, resultou em um aumento significativo de produtividade em diâmetro de copa de planta. Nesta mesma linha de pesquisa, Bündschen et al., (2018b) trabalharam com dois adubos foliares e aplicou na rúcula à campo, tendo como resultado um incremento na produtividade em número de folhas. Esses dados corroboram os resultados desta pesquisa (Quadro1).

Reforçando os dados Lopes e Guidolin (1989), mostraram que as Olerícolas, podem ter aumento na produção com a aplicação de adubação foliar, além de reduzir as deficiências nutricionais específicas.

Conforme Quadro 1, tanto o valor de MFPA e MFR tiveram o tratamento o melhor resultado em relação a testemunha, com uma diferença de 1,47 e 1,44 $\mathrm{g} \mathrm{pl}^{-1}$, respectivamente. Bandeira et al. (2011), esclarecem que um aumento de produtividade na alface pode estar diretamente ligado a aplicações de matéria-orgânica e nitrogênio, onde uma das características do ZEBMAX ${ }^{\circledR}$ é possuir uma porcentagem de nitrogênio líquido.

Resende et al. (2005) apresentou resultados satisfatórios na aplicação de $\mathrm{N}$ na cultura da alface em trabalhos à campo, sem uso de estufas, reforçando que este nutriente é extremamente importante para crescimento e desenvolvimento de folhas e está diretamente relacionada com a produção de clorofilas.

A Figura 2, apresenta os gráficos dos efeitos individuais da MFPA e MFR com a aplicação do fertilizante na alface. 
Figura 2 - Gráfico dos efeitos individuais da aplicação do fertilizante na alface $(\alpha=0,05)$ na MFPA (a). Gráfico dos efeitos individuais da aplicação do fertilizante na alface $(\alpha=0,05)$ na MFR (b)

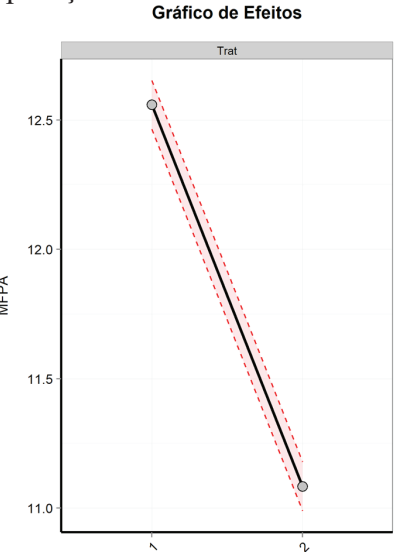

(a)

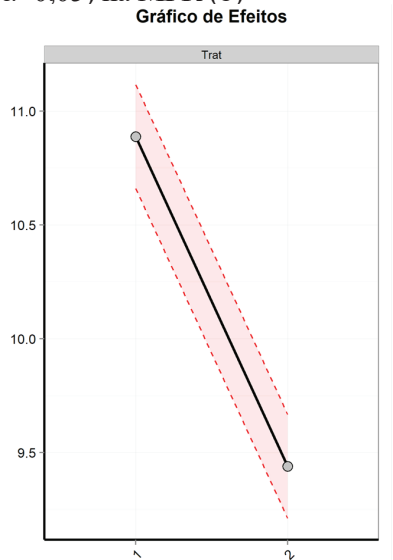

(d)
Fonte: Dados da pesquisa

Em ambas as duas Figuras (2a e 2b) mostram uma similaridade de resultados entre as análises, pois tanto no $2 \mathrm{a}$ quanto no $2 \mathrm{~b}$, quando se aplicou o $\mathrm{ZEBMAX}{ }^{\circledR}$ na alface, a resposta (MFPA e MFR), apresentaram um aumento nos dados de resposta do tratamento em relação a testemunha. Vieira e Epprecht (2009) reforçam que a aplicação de um teste com gráficos de efeitos em uma experimentação é de suma importância para observar as mudanças físicas da estrutura do experimento entre o tratamento e a testemunha.

Além dos gráficos de efeitos, os gráficos de resíduos apresentam grande importância para reforçar os resultados e dar maior confiabilidade aos dados finais. A Figura 3 apresenta os gráficos de resíduos da MFPA, sendo o histograma geral (a), o QQ-plot (b), resíduos x valores ajustados (c) e resíduos padronizados versus ordem de coleta $(d)$.

Figura 3 - Gráficos dos resíduos. Figuras 1a e 2a: Histogramas dos resíduos. Figuras 1b e 2b: QQ-plot. Figuras 1c e 2c: Resíduos versus valores preditos. Figuras $1 \mathrm{~d}$ e $2 \mathrm{~d}$ : resíduos padronizados versus ordem de coleta $(\alpha=0,05)$ para MFPA e MFR.
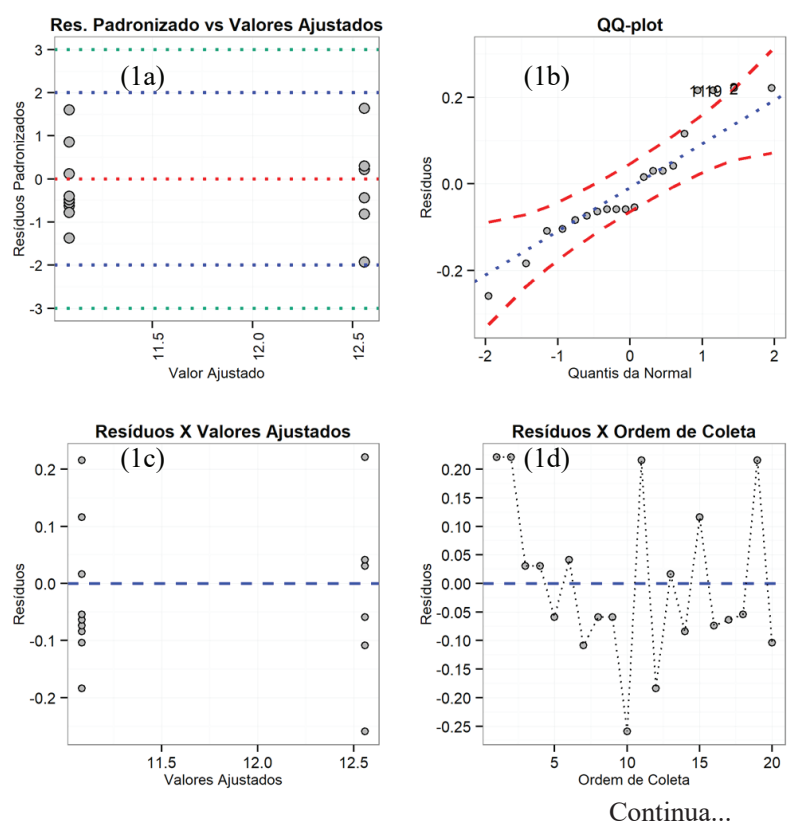

Continuação...
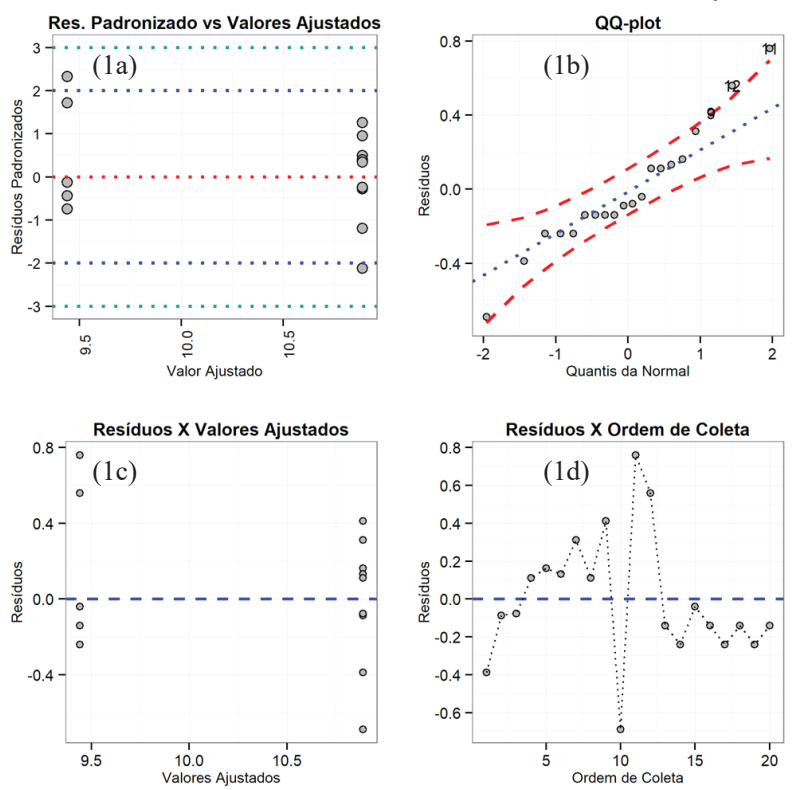

Fonte: Dados da pesquisa

Nos gráficos de resíduos nas Figuras 3.1a observa-se a distribuição aleatória dos resíduos em torno do zero, bem como na ordem de coleta dos ensaios na Figura 3.1d. Verificase também que no gráfico da probabilidade normal dos resíduos, já na Figura 3.1b, não há a presença de outliers e que os resíduos aderem a uma distribuição normal, com p-valor $=0,4113(>0,05)$ determinado pelo teste de normalidade de Anderson-Darling (AD). Essas observações quanto aos resíduos na ANOVA satisfazem a premissa da Estatística paramétrica e permite uma análise mais aprofundada dos dados.

Nos gráficos de resíduos nas Figura 3.2a observa-se a distribuição aleatória dos resíduos próximo do zero, bem como na ordem de coleta dos ensaios na Figura 3.2d. Verificase também que no gráfico da probabilidade normal dos resíduos, já na Figura 3.2b, não há a presença de outliers e que os resíduos aderem a uma distribuição normal, com p-valor $=0,4582(>0,05)$ determinado pelo teste de normalidade de Anderson-Darling (AD). Essas observações quanto aos resíduos na ANOVA satisfazem a premissa da Estatística paramétrica e permite uma análise mais aprofundada dos dados.

Como os p-valores são maiores que 5\% (MFPA 0,4113 e MFR 0,4582) para todos os testes, aceitamos a hipótese de normalidade, assim, com nível de confiança de 95\%, temos evidências de que os dados seguem uma distribuição normal.

\section{Conclusão}

Os ganhos de produtividade podem ser proporcionais aos ganhos de massa fresca da parte aérea e a massa fresca da raiz. Com isso o cultivo da alface apresentou diferenças significativas para as variáveis de crescimento (raiz e da parte aérea), em relação a aplicação do ZEBMAX® como fonte de adubação, na relação entre o tratamento e a testemunha. 


\section{Referências}

BANDEIRA, G. R. L. et al. Manejo de irrigação para cultivo de alface em ambiente protegido. Horticultura Brasileira, v. 29, p. 237-241. 2011. doi: 10.1590/S0102-05362011000200018.

BLAT, S. F. et al. Desempenho de cultivares de alface crespa em dois ambientes de cultivo em sistema hidropônico. Horticultura Brasileira, v. 29, p. 135-138, 2011. doi: 10.1590/S010205362011000100024 .

BRASILHORTIFRUTI - HFBRASIL, 2019. Anuário 2018/2019 - Retrospectiva 2018 e Perspectiva 2019, 23p. Disponível em: https://www.hfbrasil.org.br/br/revista/acessar/completo/ anuario-2018-2019.aspx. Acesso em: 15 jul. 2020.

BRASILHORTIFRUTI - HFBRASIL, 2020. O setor está preparado para resistir aos danos da COVID-19. Disponível em: https://www.hfbrasil.org.br/br/revista/o-setor-esta-preparadopara-resistir-aos-danos-da-covid-19.aspx. Acesso em: 15 jul. 2020.

BÜNDSCHEN, G. C. et al. O uso de adubo foliar após a germinação da rúcula em bandeja. In: Congresso de Ciências Agrárias da Unioeste: ciência e pesquisa para a evolução tecnológica da agropecuária - SECIAGRA, IX, 2018, Marechal Cândido Rondon. Seminário... Registro 427: livro 009, 2018 a. $831 \mathrm{p}$.

BÜNDSCHEN, G. C. et al. Desenvolvimento da rúcula nas bandejas em diferentes doses adubos foliares após a semeadura. In: Congresso de Ciências Agrárias da Unioeste: ciência e pesquisa para a evolução tecnológica da agropecuária - SECIAGRA, IX, 2018, Marechal Candido Rondon. Seminário... Registro 427: livro 009, 2018b. 831p.

FERREIRA, P.V. Estatistica Experimental Aplicada as Ciências Agrárias, Viçosa-MG, Ed. UFV, 2018.

GODINHO, E. Z. et al. Resposta da beterraba à aplicação de fertilizante mineral e organomineral no cultivo de verão. Revista Agropampa, v. 2, n. 2, 2019.

LOPES, A. S.; GUIDOLIN, J. A. Adubação Foliar. Campinas: IAC. 145 p., 1989.

MENEZES JÚNIOR, F. O. G. de; MARTINS, S. R.; FERNANDES, H. S. Crescimento e avaliação nutricional da alface cultivada em "NFT" com soluções nutritivas de origem química e orgânica. Horticultura Brasileira, v. 22, n. 3, p. $632-$ 637, 2004. doi: 10.1590/S0102-05362004000300027.

RESENDE, G. M. et al. Efeitos de tipos de bandejas e idade de transplantio de mudas sobre o desenvolvimento e produtividade de alface americana. Horticultura Brasileira, v. 21, p. 558-563, 2003. doi: 10.1590/S0102-05362003000300029.

RESENDE, G. M. et al. Produtividade e qualidade pós-colheita da alface americana em função de doses de nitrogênio e molibdênio. Horticultura Brasileira, v. 23, n. 4, p. 976-981, 2005. doi: 10.1590/S0102-05362005000400023.

RESENDE, G. M. et al. Doses de nitrogênio e molibdênio no rendimento e teor de micronutrientes em alface americana. Horticultura Brasileira, v. 28, n. 3, p. 266-270, 2010. doi: 10.1590/S0102-05362010000300004.

SALA, F. C.; COSTA, C. P. da. Retrospectiva e tendência da alfacicultura brasileira. Horticultura Brasileira, v. 30, n. 2, p. 187-194, 2012. doi: 10.1590/S0102-05362012000200002.

VIEIRA, A. F. de C.; EPPRECHT, E. K. Métodos de identificação de efeitos na dispersão em experimentos fatoriais não replicados. Gestão \& Produção, v. 16, n. 1, p. 99-110, 2009. doi: 10.1590/ S0104-530X2009000100010.

ZIECH, A. R. D. et al. Cultivo de alface em diferentes manejos de cobertura do solo e fontes de adubação. Revista Brasileira de Engenharia Agrícola e Ambiental, v. 18, n. 9, p. 948-954. doi: 10.1590/1807-1929/agriambi.v18n09p948-954. 